Vietnam Journal of Mechanics, VAST, Vol. 27, No. 1 (2005), pp. $41-50$

\title{
DAMPING IDENTIFICATION IN MULTI-DEGREE-OF-FREEDOM SYSTEMS USING THE CONTINUOUS WAVELET TRANSFORM
}

\author{
NGUYen Phong Dien \\ Hanoi University of Technology
}

\begin{abstract}
The identification of damping in multi-degree-of-freedom vibration systems is a well-known problem and appears to be of crucial interest. Compared to an estimation of the stiffness and mass, the damping coefficient or, alternatively, damping ratio is the most difficult quantity to determine. In this paper, the continuous wavelet transform based on the Morlet-wavelet function is used to identify the modal damping ratios of multi-degree-offreedom vibration systems. A new wavelet-based method for the damping identification from measured free responses is presented. The proposed method was also tested by experiments on a steel beam.
\end{abstract}

\section{INTRODUCTION}

The identification of damping in multi-degree-of-freedom vibration systems is a wellknown problem and appears to be of crucial interest. Compared to an estimation of the stiffness and mass, the damping coefficient or, alternatively, damping ratio is the most difficult quantity to determine. While both mass and stiffness can be determined by static tests, damping requires a dynamic test to measure. The majority of damping measurements performed today are based on experimental modal analysis or modal testing [8]. However, this approach requires a specific measurement hardware and a complex software for determining the frequency response function of the system and extracting the modal data. Furthermore, the frequency response functions will often give significant errors resulting from the influence of the noise and the spectrum overlap.

Over the past 10 years, wavelet theory has become one of the emerging and fastevolving mathematical and signal processing tool for its many distinct merits. General overview of application of the wavelet transform may be found in $[2,6,7]$. The continuous wavelet transform has been proved to have a high level of efficiency in accurate information and short processing time. The wavelet transform has dominant advantages in signal filtering and other merits such as time-frequency characteristics, which make it possible to parameter identification [3-5].

In this paper, the continuous wavelet transform based on the Morlet-wavelet function is used to identify the modal damping ratios from free responses of linear vibration systems with multi-degree-of-freedom. The following examples demonstrate the improvements of the proposed wavelet-based method on the damping identification.

\section{THE CONTINUOUS WAVELET TRANSFORM}

This section presents a brief background on the Continuous Wavelet Transform (CWT) utilized in this paper. A thorough discussion of wavelets is given in references [1], [7]. 
Using a mother-wavelet function $\psi(\mathbf{t})$, wavelets are family of functions of type

$$
\psi_{\tau, s}(t)=\frac{1}{\sqrt{s}} \psi\left(\frac{t-\tau}{s}\right), \quad \tau, s \in \mathbf{R}^{+}
$$

generated from $\psi(t)$ by the operation of dilation by $s$ and translation by $\tau$. In the time domain, $\psi_{\tau, s}$ is centered at $\tau$ with a spread proportional to $s$. The CWT of a signal $x(t)$ is defined by:

$$
C W T\{x(t)\}=W_{x}(\tau, s)=\frac{1}{\sqrt{s}} \int_{-\infty}^{+\infty} x(t) \psi^{*}\left(\frac{t-\tau}{s}\right) d t
$$

where $\psi^{*}$ is the complex conjugate of the wavelet $\psi$. A wavelet coefficient $\mathrm{W}_{x}(\tau, s)$ measures the variation of signal $x(t)$ in a neighborhood of position $\tau$. By varying the scale $s$ and translating along the localized time $\tau$, the amplitude of wavelet coefficients $\left|W_{x}(\tau, s)\right|$ can construct a wavelet amplitude map showing both the amplitude of any features in the signal versus the scale and how this amplitude varies with time. Therefore, information in the time domain will still remain, in contrast to the Fourier Transform (FT), where the time domain information becomes almost invisible after the integration over entire record length of the signal.

$$
F T\{x(t)\}=\hat{x}(\omega)=\int_{-\infty}^{+\infty} x(t) e^{i \omega t} d t
$$

A complex mother-wavelet can be constructed with a frequency modulation of a real and symmetric window $g(t)$.

$$
\psi(t)=g(t) e^{i \eta_{0} t}
$$

where $\eta_{0}$ is a constant parameter and $i=\sqrt{-1}$. A Morlet mother-wavelet is obtained with a Gaussian window.

$$
\begin{gathered}
g(t)=\pi^{-1 / 4} e^{-t^{2} / 2}, \\
\hat{g}(\omega)=\pi^{-1 / 4} e^{-\omega^{2} / 2} .
\end{gathered}
$$

In this study the Morlet wavelet is used to perform the CWT. The relation between the scale $s$ and the frequency $\omega$ of the Morlet wavelet can be derived analytically as [1].

$$
s=\frac{\eta_{0}}{\omega} \text {. }
$$

As can be seen from (2.2), the CWT is a linear time-frequency transform. Its linearity makes it possible to analyze each i-th component $x_{i}(t)$ of a multi-component signal.

$$
C W T\left\{\sum_{i=1}^{n} x_{i}(t)\right\}=\sum_{i=1}^{n} C W T\left\{x_{i}(t)\right\} .
$$




\section{MODAL DAMPING RATIO IDENTIFICATION}

\subsection{Signal envelope extraction with the CWT}

Consider a signal $x(t)$ that can be expressed by a modulated sinusoidal function

$$
x(t)=a(t) \cos \left(\alpha_{0} t+\beta\right)=a(t) \cos \varphi(t),
$$

where $a(t)$ is the envelope of $x(t)$ and $\varphi(t)=\alpha_{0} t+\beta$.

The CWT of this signal can be derived analytically by a simple function as follows (see also [1])

$$
W_{x}(\tau, s)=\frac{\sqrt{s}}{2} a(\tau) e^{i \varphi(\tau)} \hat{g}\left(\eta_{0}-s \alpha_{0}\right)+\varepsilon(\tau, s)
$$

where $\hat{g}\left(\eta_{0}-s \alpha_{0}\right)$ is the Fourier transform of $g(t)$ at $\omega=\eta_{0}-s \alpha_{0}$ and $\varepsilon(\tau, s)$ is the corrective term. If the variations of $a(t)$ are slow compared to the period $2 \pi / \alpha_{0}$, the term $\varepsilon(\tau, s)$ can be neglected $[1,3]$. In this case, by considering expression (2.6) the CWT of $x(t)$ can be written in the form:

$$
W_{x}(\tau, \omega)=\frac{1}{2} \sqrt{\frac{\eta_{0}}{\omega}} a(\tau) e^{i \varphi(\tau)} \hat{g}\left(\eta_{0}-\frac{\eta_{0} \alpha_{0}}{\omega}\right) .
$$

The amplitude of wavelet coefficients $\left|W_{x}(\tau, s)\right|$ is given by

$$
\left|W_{x}(\tau, \omega)\right|=\frac{1}{2} \sqrt{\frac{\eta_{0}}{\omega}} a(\tau)\left|\hat{g}\left(\eta_{0}-\frac{\eta_{0} \alpha_{0}}{\omega}\right)\right| .
$$

Since $|\hat{g}(\omega)|$ is maximum at $\omega=0$, expression (3.4) shows that $\left|W_{x}(\tau, \omega)\right|$ is maximum at $\omega=\alpha_{0}$. For $\omega=0$ expression (2.5b) becomes $\hat{g}(0)=\pi^{-1 / 4}$. The envelope $a(\tau)$ at $\omega=\alpha_{0}$ is given by

$$
a(\tau)=2 \pi^{1 / 4} \sqrt{\alpha_{0} / \eta_{0}}\left|W_{x}\left(\tau, \alpha_{0}\right)\right|=\mathrm{K} .\left|W_{x}\left(\tau, \alpha_{0}\right)\right|,
$$

where $K$ is a positive constant $K=2 \pi^{1 / 4} \sqrt{\alpha_{0} / \eta_{0}}$.

Expression (3.5) shows that the wavelet coefficients $\left|W_{x}(\tau, \omega)\right|$ at $\omega=\alpha_{0}$ (called as wavelet envelope [5]) is proportional to the envelope of signal $x(t)$ described in (3.1).

\subsection{Damping ratio estimation using CWT}

It is well known that a damped $n$ - degree-of-freedom system with proportional damping

$$
\mathbf{M} \ddot{\mathbf{q}}+B \dot{\mathbf{q}}+C \mathbf{q}=0,
$$

can be solved by using the modal analysis [8]. This leads on $n$ decoupled modal equations

$$
\ddot{p}_{i}(t)+2 \zeta_{i} \omega_{0 i} \dot{p}_{i}(t)+\omega_{0 i}^{2} p_{i}(t)=0, i=1,2, \ldots, n
$$

Here $p_{i}(t)$ denotes the $i-$ th modal coordinate, $\omega_{0 i}$ is the $i$-th undamped natural frequency and $\zeta_{i}$ is the $i$-th modal damping ratio. The modal damping places an energy dissipation term of the form $2 \zeta_{i} \omega_{0 i} \dot{p}_{i}(t)$ (viscous damping). This form is chosen largely for its mathematical convenience. The modal damping ratios $\zeta_{i}$ are assigned by making measurements of the free damped response and estimating $\zeta_{i}$. 
The task of interest is to determine the modal damping ratios associated with each mode shape. The linearity of the CWT is useful for extracting modal data of each mode from measured free responses of the system. This will be demonstrated in section 4 .

Consider the free response of a linear vibration system for the unterdamped case, the signal model corresponding to the $i$-th mode can be described by

$$
x_{i}(t)=A_{i} e^{-\zeta_{i} \omega_{0 i} t} \sin \left(\omega_{d i} t+\phi_{i}\right),
$$

where amplitude $A_{i}$ is a constant and $\omega_{d i}$ is the $i$-th damped natural frequency The decay envelope of this signal is $a_{i}(t)=A_{i} e^{-\zeta_{i} \omega_{0 i} t}$. Note that it is difficult to extract the envelope $a_{i}(t)$ from measured free response with numerous natural frequencies. According to the signal model (3.8), the logarithmic decrement $\delta_{i}$ is given by

$$
\delta_{i}=\frac{1}{m} \ln \frac{a_{i}(t)}{a_{i}\left(t+m T_{i}\right)},
$$

where $m$ is any positive integer and $T_{i}=2 \pi / \omega_{d i}$. Since the variations of $a_{i}(t)$ are slow compared to the period $T_{i}$, using expressions (3.5) and (3.9) the logarithmic decrement $\delta_{i}$ can be expressed in term of wavelet coefficients as

$$
\delta_{i}=\frac{1}{m} \ln \frac{\mid W_{x i}\left(t, \omega_{d i} \mid\right.}{\left|W_{x i}\left(t+m T_{i}, \omega_{d i}\right)\right|} .
$$

Finally, the modal damping ratio can be determined from logarithmic decrement $\delta_{i}$ [8].

$$
\zeta_{i}=\frac{\delta_{i}}{\sqrt{4 \pi^{2}+\delta_{i}^{2}}} .
$$

In summary, the following procedure is required for identifying damping ratios:

- Detecting local maxima in the wavelet amplitude map to determine natural frequencies.

- Extracting the wavelet envelope $\left|W_{x}(\tau, \omega)\right|$ at the natural frequencies.

- Calculating the damping ratios (Formulas (3.10) and (3.11)).

A specialized program has been developed on the MATLAB ${ }^{\circledR}$ numeric computing environment for this study. The program includes two modules:

- Signal processing functions for the continuous Wavelet-Transform.

- Calculating the damping ratios according to the above-mentioned procedure.

\section{NUMERICAL EXAMPLE}

In order to assess the performance of the proposed wavelet-based method, a testsignal with three exponentially-decaying components has been chosen for simulating the free response of a damped vibration system.

$$
x(t)=\sum_{i=1}^{3} a_{i} e^{-\zeta_{i} \omega_{0 i} t} \sin \left(\omega_{d i} t+\phi_{i}\right) .
$$


Table 1. Parameters of the test-signal

\begin{tabular}{|c|c|c|c|}
\hline Component $i$ & 1 & 2 & 3 \\
\hline$f_{i}=\omega_{d i} / 2 \pi(\mathrm{Hz})$ & 250 & 500 & 1250 \\
\hline$\zeta_{i}$ & 0.02 & 0.045 & 0.005 \\
\hline$\phi_{i}$ & $-\pi / 8$ & $\pi / 6$ & $\pi / 8$ \\
\hline$a_{i}$ & 1.0 & 1.25 & 0.5 \\
\hline
\end{tabular}

Fig. 1 shows the signal in the time domain. The parameters of the signal are given in Table 1. The frequencies of signal-components are different, the last component has a high frequency with a small damping ratio.

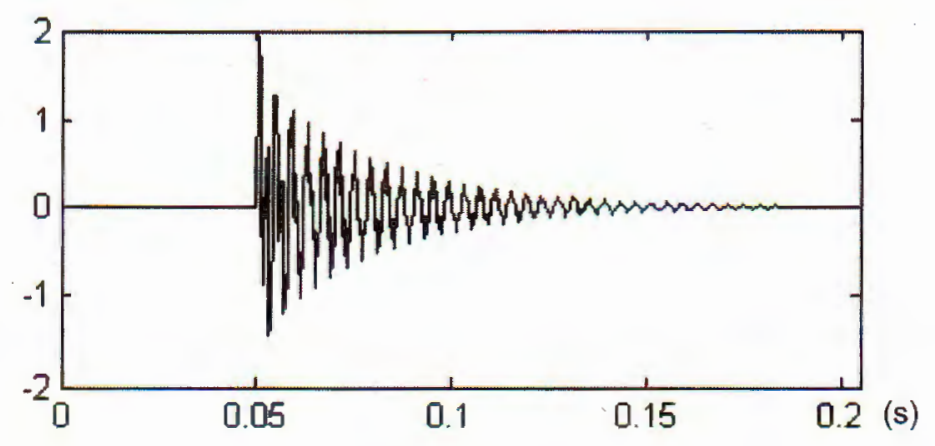

Fig. 1. The test-signal

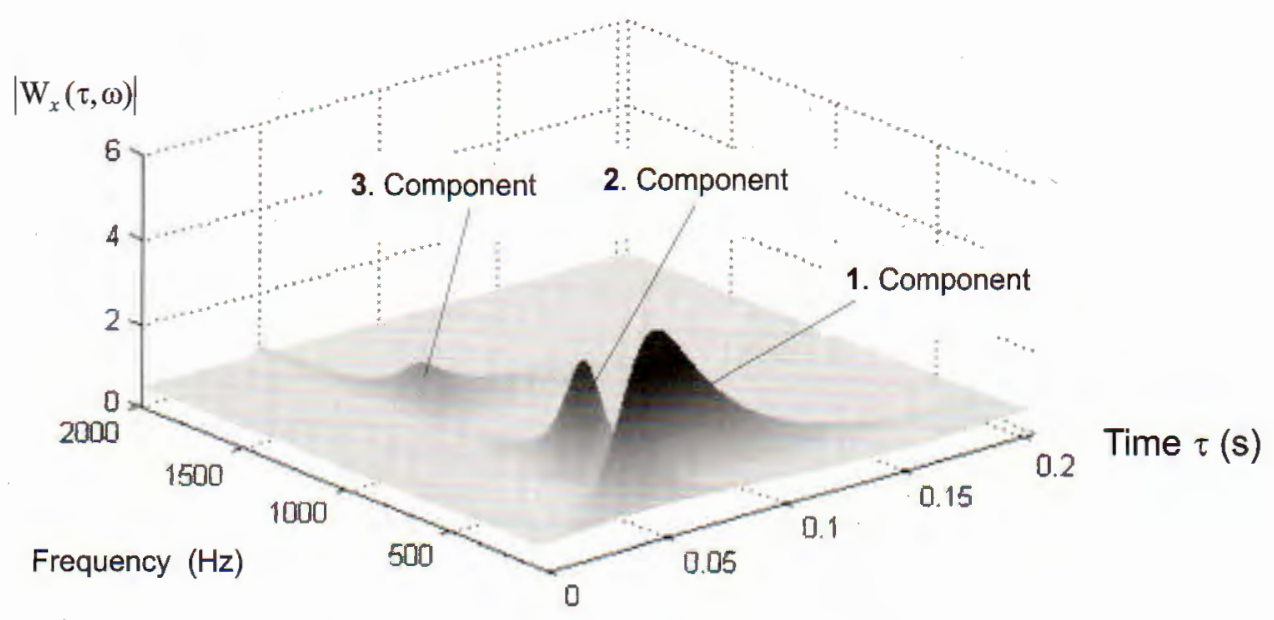

Fig. 2. The wavelet amplitude map of the test-signal

Firstly, the signal is transformed in time-frequency domain using the CWT with Morlet-wavelet. Fig. 2 is the wavelet amplitude map displayed as a three-dimensional surface, obtained by plotting the amplitude of the wavelet coefficients $\left|W_{x}(\tau, \omega)\right|$ against 
time and frequency. Three exponentially-decaying components are separated from the original signal and can be clearly identified.

In order to identify "natural" frequency $\omega_{d i}$ of each signal-component from the map in Fig. 2, a numerical algorithm is developed to seek local maxima of the three-dimensional surface [4]. The positions of the local maxima reveal the corresponding natural frequencies in frequency axis (see section 3.1).

The wavelet envelope $\left|W_{x}\left(\tau, \omega_{d i}\right)\right|$ can be extracted from the wavelet amplitude map by a slice parallel to the time axis through frequency $\omega_{d i}$ in the frequency axis. According to formulas (3.10) and (3.11), this envelope can be used to determine the damping ratio of signal components. Fig. 3 shows the envelope extracted at the third natural frequency.

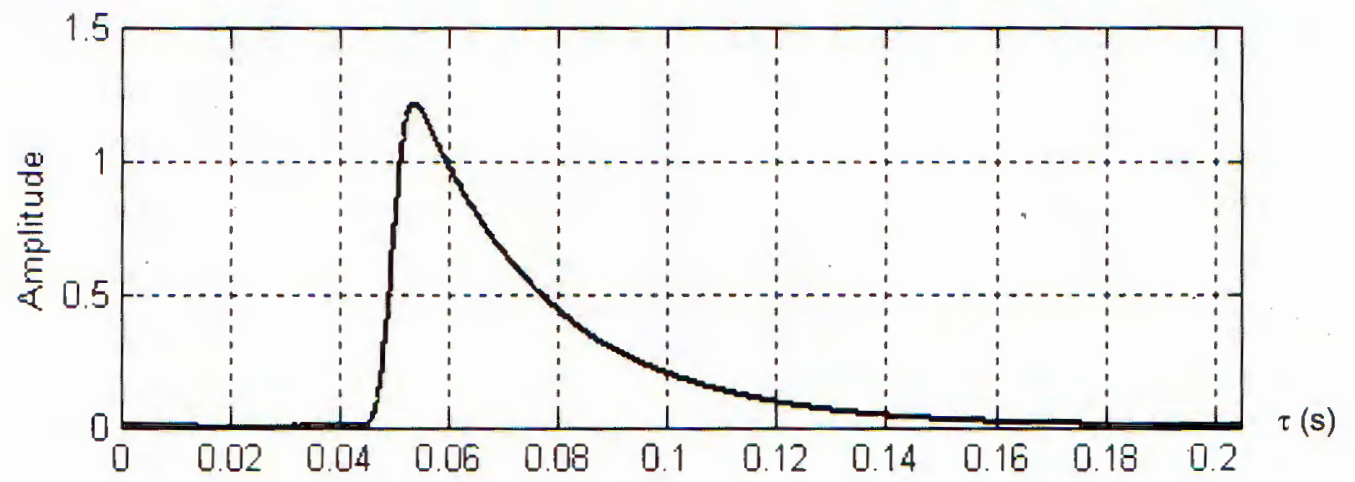

Fig. 3. The wavelet envelope of the third signal-component extracted from the map in Fig. 2

Table 2. Results of damping identification for the test-signal

\begin{tabular}{|c|c|c|c|}
\hline Parameter & $i=1$ & $i=2$ & $i=3$ \\
\hline$\omega_{d i} / 2 \pi(\mathrm{Hz})$ & $\mathbf{2 4 7}$ & $\mathbf{5 0 0}$ & $\mathbf{1 2 5 5}$ \\
\hline$T_{i}(\mathrm{~s})$ & $4 \times 10^{-3}$ & $2 \times 10^{-3}$ & $7.97 \times 10^{-4}$ \\
\hline$m$ & 10 & 15 & 50 \\
\hline$\tau_{0}$ & 0.080 & 0.0567 & 0.060 \\
\hline$\left|W_{x}\left(\tau_{0}, \omega_{d i}\right)\right|$ & 2.6167 & 2.5620 & 0.9835 \\
\hline$\left|W_{x}\left(\tau_{0}+m T_{i}, \omega_{d i}\right)\right|$ & 0.7340 & 0.0485 & 0.2060 \\
\hline$\delta_{i}$ & 0.1271 & 0.2645 & 0.0313 \\
\hline$\zeta_{i}$ & $\mathbf{0 . 0 2 0 2}$ & $\mathbf{0 . 0 4 2 1}$ & $\mathbf{0 . 0 0 5}$ \\
\hline Error of $\omega_{d i}(\%)$ & 1.1 & 0.0 & 0.4 \\
\hline Error of $\zeta_{i}(\%)$ & 1.0 & 6.4 & 0.0 \\
\hline
\end{tabular}

The identification data is given in Table 2. It can be seen that the identification of the natural frequencies and the damping ratios is very good.

\section{EXPERIMENT}

Analysis of the measured impulse response of a beam provides a good demonstration of the proposed wavelet-based method. The experiment was done at a uniform steel beam of 
rectangular cross-section $(25 \times 30 \mathrm{~mm})$ with the clamped-clamped boundary configuration (Fig. 4).

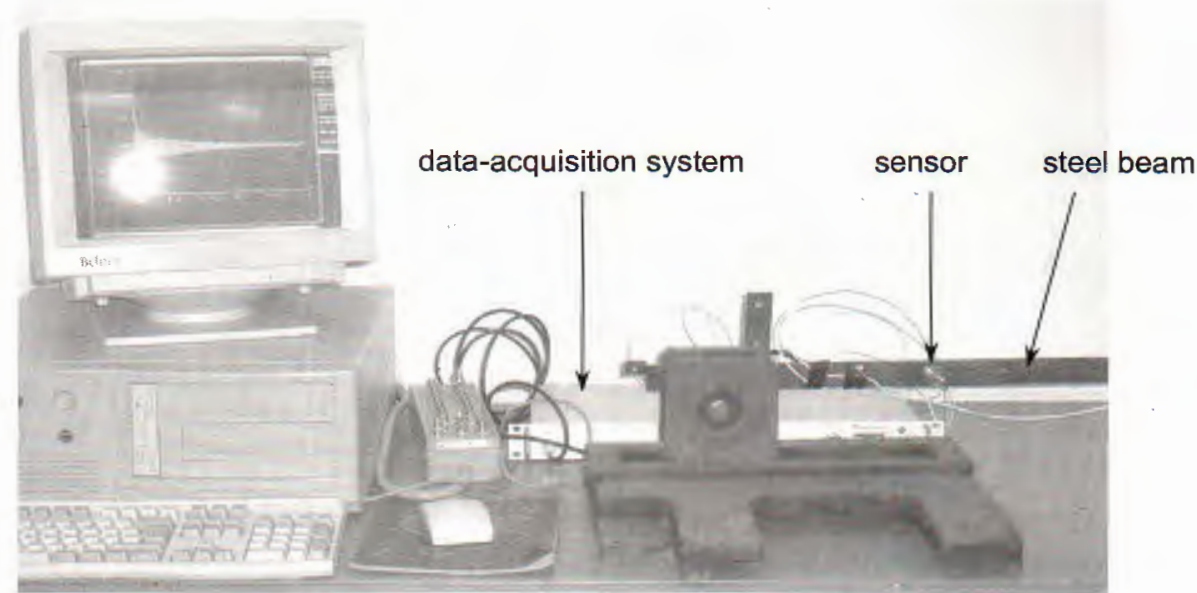

Fig.4. Experimental set-up

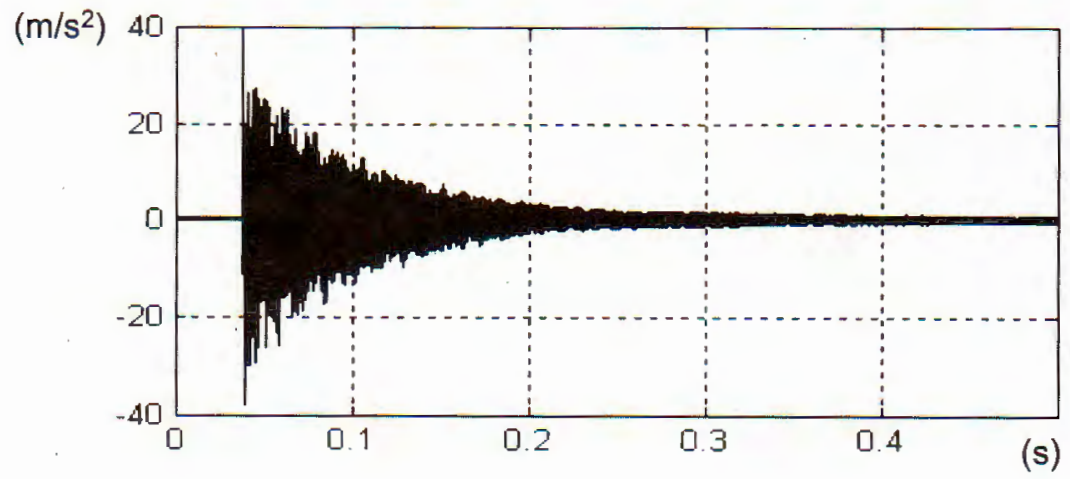

Fig. 5. Measured vibration response of the beam: time record

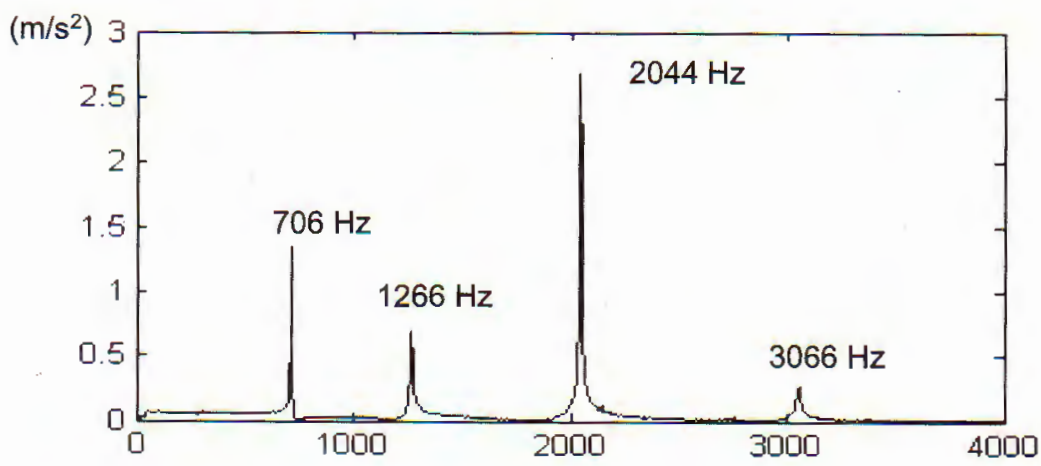

$(\mathrm{Hz})$

Fig.6. Measured vibration response of the beam: frequency spectrum 


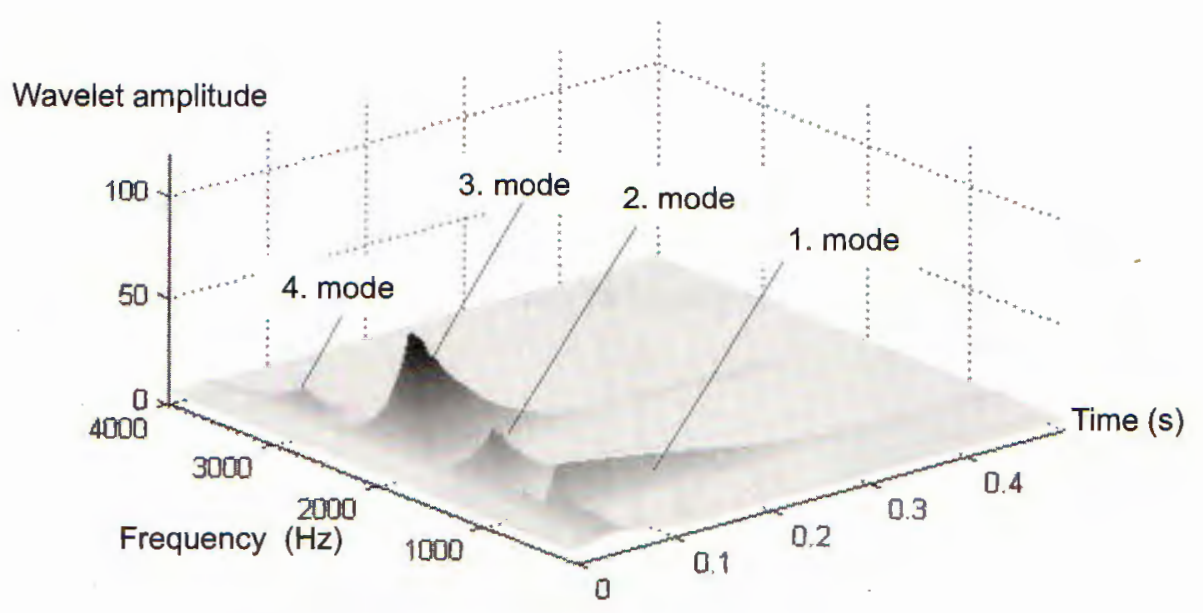

Fig. 7. The wavelet amplitude map of the measured vibration signal shown in Fig. 5

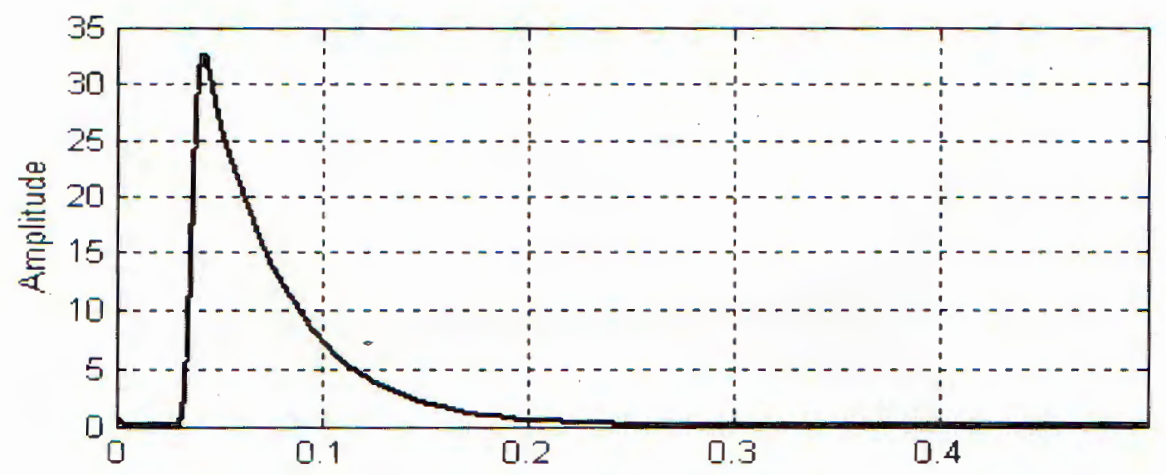

Fig. 8. The wavelet envelope at the $2^{\text {nd }}$ natural frequency

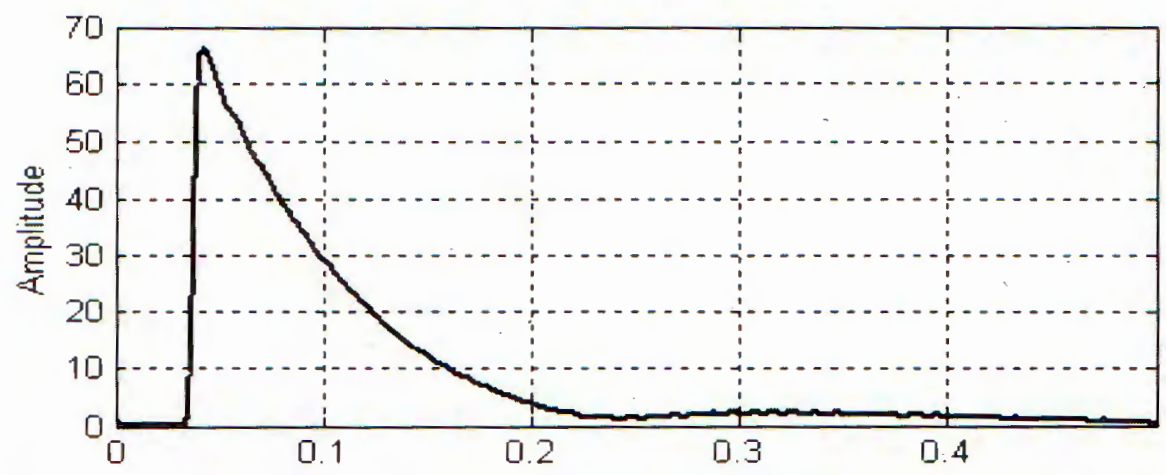

Fig. 9. The wavelet envelope at the $3^{\text {rd }}$ natural frequency 
A hammer with a hard tip and a soft tip was used for generating impacts. An accelerometer on the beam at the closest position to the point of the impact measured response of bending vibration of the beam. The vibration signals were sampled at the sampling frequency of $10 \mathrm{kHz}$ by a multi-channel data-acquisition system.

The time record of the vibration response is shown in Fig. 5. The natural frequencies of the beam can be determined in advance by means of the spectral analysis with FFT. Fig. 6 displays the spectrum with the first four natural frequencies.

Since the acceleration of the free response at each vibration mode is nearly proportional to the corresponding displacement [3], the measured acceleration signal can be directly used for damping identification. Fig. 7 displays the wavelet amplitude map of the measured signal. The wavelet envelopes displayed in Figs. 8 and 9 were used for estimating the damping ratios.

Table 3. Results of damping identification

\begin{tabular}{|c|c|c|c|}
\hline \multirow{2}{*}{ Mode } & \multicolumn{2}{|c|}{ Natural frequencies $[\mathrm{Hz}]$} & \multirow{2}{*}{} \\
\cline { 2 - 3 } & FFT-method & CWT-method & \multicolumn{1}{|c|}{} \\
\hline Mode 1 & 706 & 707 & 0.0015 \\
\hline Mode 2 & 1266 & 1269 & 0.0032 \\
\hline Mode 3 & 2044 & 2044 & 0.0012 \\
\hline Mode 4 & 3066 & 3084 & 0.0041 \\
\hline
\end{tabular}

The main results of the damping identification for the beam are given in Table 3 . Note that the damping ratio $\zeta_{i}$ of the bending vibration of steel is small unless the structure contains viscoelastic material or a hydraulic damper is present. Common values are expected to be in the range $0 \leq \zeta_{i} \leq 0.05$ (reference [8]). Therefore, the results seem to be in good agreement.

\section{CONCLUSIONS}

A wavelet-based method for the damping identification from measured free response of multi-degree-of-freedom vibration systems is presented. The proposed method carries out the continuous wavelet transform of the response and the extraction wavelet envelopes in time-frequency domain. The damping ratio of each vibration mode is then determined by the value of the logarithmic decrement.

The wavelet-based method was also tested by experiments on a steel beam. As can be seen from the identification data, this innovative approach proves to be a very effective signal processing tool for the experimental vibration analysis.

Acknowledgment. This paper was completed with the financial support of the Vietnam Basic Research Program in Natural Science.

\section{REFERENCES}

1. S. Mallat, A Wavelet Tour of Signal Processing, Academic Press, San Diego, London, New York, 1999.

2. Z. K. Peng, F. L. Chu, Application of the wavelet transform in machine condition monitoring and fault diagnostics: a review with bibliography, Mechanical Systems and Signal Processing 18 (2004) 199-221. 
3. J. Slavic, I. Simonovski, M. Boltezar, Damping identification using a continuous wavelet transform: application to real data. Journal of Sound and Vibration 262 (2003) 291-307.

4. D. E. Newland, Ridge and Phase Identification in the Frequency Analysis of Transient Signals by Harmonic Wavelets, ASME Journal of Vibration and Acoustics 121 (1999) 149-155.

5. E. L. Schukin, R. U. Zamaraev, L. I. Schukin, The optimisation of wavelet transform for the impulse analysis in vibration signals. Mechanical Systems and Signal Processing 18 (2004) 1315-1333.

6. G. Meltzer, Nguyen Phong Dien, Fault diagnosis in gears operating under non-stationary rotational speed using polar wavelet amplitude maps. Mechanical Systems and Signal Processing 18 (2004) 985-992.

7. Nguyen Phong Dien, Beitrag Zur Diagnostik Der Verzahnungen in Getrieben Mittels ZeitFrequenz-Analyse, Fortschritt-Berichte VDI, Reihe 11, Nr. 135, VDI-Verlag, Dueselldorf, 2003.

8. D. J. Inman, Engineering Vibration, Prentice-Hall Publisher, New Jersey, 2001.

Received September 6, 2004

Revised November 24, 2004

\section{NHẬN DẠNG TY̛ SỐ CẢN CƯA HỆ NHIỀU BẬC TỰ DO BẰNG PHÉP BIẾN DỔI WAVELET LIÊN TỤC}

Việc nhận dạng cản trong hệ dao động nhiều bậc tự do là chủ đề quen thuộc và ngày càng nhận được nhiều sự quan tâm. So với việc đánh giá các đại lương độ cứng và khối lượng, các hệ số cản hoặc tỷ số cản là đại lượng khó xác định nhất. Trong bài báo này, phép biến đảioi Wavelet liên tục trên cơ sở hàm Morlet-wavelet được áp dụng để nhận dạng tỳ số cản của hệ dao động nhiều bậc tụ̣ do. Bài báo trình bày một phương pháp mới trên cơ sở biến đổi wavelet để nhận dạng cản từ các đáp ứng của hệ đo được. Phương pháp này đã được kiểm chứng thông qua các thực nghiệm trên một dầm thép. 